\title{
Perceived Stress, Positive-Negative Emotions, Personal Values and Perceived Social Support in Malaysian Undergraduate Students
}

\author{
Siamak Khodarahimi ${ }^{1, *}$, Intan H.M. Hashim ${ }^{2}$, Norzarina Mohd-Zaharim ${ }^{2}$ \\ ${ }^{1}$ Department of Psychology, Eghlid Branch, Islamic Azad University, Eghlid, Iran \\ ${ }^{2}$ Department of Psychology, School of Social Sciences, Universiti Sains Malaysia, Penang, Malaysia
}

\begin{abstract}
The purpose of this research was to examine the perceived stress, emotion regulation, positive-negative emotions, personal values and perceived social support and also to investigate the effects of gender, ethnicity and religion on these constructs. Participants were 308 students from a public university in Malaysia. A demographic questionnaire and four self-report inventories were used in this study. Results showed that perceived stress and its subscales had significantly positive correlations with both positive and negative emotions. The perceived stress and negative life events hassles had significantly negative relationships with personal values and its subscales. Even so, there are significant negative relationships between the perceived stress and personal hassles with perceived social support and its family and friend subscales. Findings rejected the effects of gender and marital status in perceived stress but affirmed the roles of religion and ethnicity on it. The negative emotions and perceived social support explained 37 and 39 percents of the perceived stress variation in total sample and females respectively, and negative emotions explained 48 percent of perceived stress among males.
\end{abstract}

Keywords Perceived Stress, Positive-Negative Emotions, Personal Values, Perceived Social Support

\section{Introduction}

Stress is a highly unpleasant state of emotional arousal that humans experience in settings perceived as treacherous and intimidating. Individuals differ in their capability to experience stress and in their ability to coping with it efficiently but perpetual and boring stress levels can result to break down. High and chronic stress with poor coping resources might as well produce psychopathological reactions, which termed anxiety disorders (1-3). Stress develops from any interaction between an individual and the environment that the individual recognizes the situation as threatening, challenging or possibly harmful. Really, stress is an external constraint that directly upsets the individual both mentally and physically. Individual in a stressful situation will be influenced in his or her mental ability to carry out for ongoing tasks. Auerbach proposed that stressors embrace those circumstances that stress-evoking, and commonly are classified into three general categories: Catastrophic events, major life changes, and daily hassles (2). Lazarus and Folkman suggested the transactional nature of stress that in literature often called psychological stress and cognitive

* Corresponding author:

khodarahimi@yahoo.com (Siamak Khodarahimi)

Published online at http://journal.sapub.org/ijpbs

Copyright (C) 2012 Scientific \& Academic Publishing. All Rights Reserved appraisal theory (4). They suggested that stress is a two way process; the environment provokes stressors, and in return an individual looks for ways to encounter with these. Thus, stress is social realty that might masteries the individual in various groups.

\subsection{Stress in Students}

Likewise, stress in college students is an expanding phenomenon (5). Kari and Heiman indicated numerous Stressors during the term-time, containing academic interrogates and social adaptation in the university students (6). Bazeley and Thyer asserted that the majority of stressors that felt by the students inevitably resulted from anxiety related to educational programs like exams, passing assignments, etc (7). Mahat reflected the majority of the students reported the interpersonal relationships as an extremely stressful interaction with their teachers (8). Kirland supported priority stressors in university students to include in descending: order: Academic, environmental, financial, interpersonal, and personal (9). Irani affirmed that stress is a factor in university students' for choice of their major (10). Kirkland indicated that some ethnic university students show a more stress than others, and this is more prevalent in students who might come to college from lower-income families, and often they may be less prepared academically (9). Research revealed that race, ethnicity, and gender constructs could influence perceived stress in university students $(11,12)$. 
Also, research among university students revealed that there is a significant difference between male and female students, with females experiencing more stress (13). Alternatively, students in second year or more had the highest level of stress (14). In sum, university students stress is result of their adaptation to multiple changes in university and its surrounding atmosphere, that have four sources including: the environmental (weather, pollution, and noise), social (examinations, responsibilities, family, friends, classmates, and roommates), physiological (illness, menopause, injuries, and nutrition), and cognitive (perfect performance and the interpretation of others' reactions). Hudd and colleagues found that university students high stress levels will result to more unhealthy behaviors among them (15). Sarafino and Ewing emphasize that reduce stress is of paramount importance to university counseling centers because of its adverse outcomes in university students (16). But any effective program and intervention in students' stress mostly depend to examination the roles of personal psychological resources on their perceived stress.

Now, this study define stress as a real or perceived imbalance between environmental demands required for survival and an individual's capacity to adapt to these requests, and this definition based to the previous stress conceptualizations by Lazarus and Folkman, Chrousos and Gold, Lovallo and Auerbach (1,4,17-19). Moreover, Lazarus conceptualized the main components of the stress process, including environmental demands, subjective evaluations of stressful situations, and stress-related emotional responses (17). Present conceptualization considers the stress as part of a sequential process in which objective environmental circumstances are appraised by the individuals. The subjective or perceived stress of multiple stressful events can present a potential threat, danger, change or challenge to one's well-being or survival. Then perceived stress is often reflecting in changes in outward behavior and boredom or other emotional states. Thereby, it suggested that individual perceived stress in university students would have relationships with positive-negative emotions, personal values, social support, and interpersonal relationships as their psychological resources among them. Plausible relationship between stress and emotions types is somewhat new area in psychological research.

\subsection{Stress and Emotional Problems}

Murberg and Bru proved that stressors relate with emotional problems (20). Khodarahimi, Rasti, Khajahe and Sattar reported a few of emotional problems in university students, including depression, anxiety, and aggression (21). Hereby, it strongly advocated that positive and negative types of emotions have a significant role in students' perceived stress. Recent literature and conceptualization have shown positive and negative emotions to be linked with mental health (22). Salmela proposed a two-levels account of the emotional affectivity that explain how negative and positive emotions play possible roles in adaptation against daily problems (23). Negative emotions' examples are: apathy, grief, fear, hatred, shame, blame, regret, resentment, anger, hostility. Positive emotions' examples are: interest, enthusiasm, boredom, laughter, empathy, action, curiosity. Both negative and positive emotions are unique and special adaptations in the scheme of human evolution, and argued that they are markers of illness and well-being, and that they trigger approach behavior. It reflected that all positive emotions are merely outsets to any negative emotions to make life more enrich. Unfortunately, in existent taxonomy of basic emotions across cultures and languages, some researchers typically identify only one positive emotion for every three or four negative emotions (24). These positive and negative emotions spotlight the role of specific action tendencies that emphasize the functions of emotions as evolved adaptations $(22,25)$. However, experiencing a positive emotion leads to states of mind and to modes of behavior that prepare an individual for later hard times. In broaden-and-build theory, Fredrickson proposed that the positive emotions broaden an individual's momentary mindset, and they are useful to build enduring personal coping resources (22). Altogether, in part with Fredrickson this study hinted negative and positive emotions affect the students' perception models toward their stressors and how to cope against them.

\subsection{Stress and Personal Values}

In fact, any instruction is a value-bounded process, and this is right for all higher education systems and students' life in campuses. Personal values might encompass elements from preceding values' theories (26-29)). According to these theories, values show a key role in shaping all attitudes and behaviors. Values appear for one of the most crucial factors that contributing to cultural effects at personal and collective levels. It suggested that through the practice of daily experience values become defined in terms of the kind of general goal it represents and assumes a place on the scale of an individual's value priorities. According to Kahle, values can be grouped into the internal and external locus of control, which is referred to as the degree to which a person feels that he/she has control over the surroundings around them and those out of their control (30). Kahle was identified six internal-oriented values and three external-oriented ones. The former included the sense of fulfillment self-respect, self-fulfillment, warm relationships with others, fun and enjoyment, and excitement while the latter comprise a sense of belonging, security, and being well-respected (30). The values that individuals believe they are those that would benefit them if acted upon. For example, individuals which claim that security are an important principle in their lives it would contribute to their attainment of group harmony and stability. However, the central role played by the personal values is exhibited in research on culture, which often begins with a set of values and assumptions (31). Therefore, it expected that personal values as an individual resource in students will influence their wide-ranging scope toward perceived stress in university. 


\subsection{Social Support and Stress}

Social support is an important constituent of college students' transitioning successfully to the college environment (32). This assumption draws attention to the idea that it might be beneficial for students to draw support from several sources rather than just relying on one (33). According to Cobb (34) social support is information leading the subject to conceive that he is cared for and loved, esteemed, valued and belongs to a network of communication. Cassel (35) defined social support with regard to the social environment and health, and particularly the protective benefits of social integration. In a broad spectrum, social support is the process by which individuals feel valued, cared for, and connected to a group of people (36).

The Perceived or functional support is a subjective judgment that family and friends would provide the quality assistance with future stressors. People with a high perceived-support often believe that they can deem on their family and friends to provide a useful help during the times of trouble (37). For attachment theory, support seeking and support provision is an essential for the survival of human beings (38). Teachers and supervisors in academic settings can as well serve as significant suppliers of support (39). All groups, institutions, and spiritual figures real or imagined can further be used as attachment figures that improve individual social support. In fact, mental images of attachment figures can serve as internal sources of support. They may also provide the internal models of supportive behavior that help a person self-soothe in the absence of physically present attachment figures $(40,41)$. Investigation on the social support has centered on identifying how power and status in network relationships reflect on the social support obtained through kinship relations and those obtained through relationships with friends, neighbors, and co-workers (42). Vaux (43) argued the links between support resources, behavior and appraisals. According to social-cognitive perspective, negative evaluations of the self, important others' people are connected together in cognitive networks and its perceived support (44). During the last two decades, a growing body of literature has sought to understand the connections between social support and well-being by examining supportive communication that means "verbal and nonverbal behavior produced with the intention of providing assistance to others perceived as needing that aid" (45). In the supportive-communication perspective, the communication of emotional support is often termed comforting, and it has a variety of forms across the life span and different aged groups (46). Dual-process approach to the social support is a current theory that argues individuals' responses to any supportive communication will depend on how the recipient attends to and thinks about the actual content of a supportive message (47). Therefore, authors suggest that perceived social as a subjective phenomenon that originated in childhood attachment and present personal appraisals endeavors in the social network would influence students' perceived stress.

\subsection{Present Study}

Accordingly, the current study founded on the confidence levels of undergraduate students, particularly that how do they perceive stress, positive and negative emotions, personal values, and social support in University Sains of Malaysia. Based on aforementioned conceptualizations to the perceived stress, positive-negative emotions, personal values, and social support, it speculated that there are relationships between these variables. The first hypothesis of this research study is that perceived stress, positive and negative emotions; personal values and social support have significant correlations in university undergraduate students. The second hypothesis of this research study is that gender, marital status, ethnicity, and religion will play significant effects in perceived stress in undergraduate students. The third hypothesis of this project is that positive and negative emotions, personal values, and social support constructs will predict the perceived stress in this sample.

\section{Method}

\subsection{Participants and Procedure}

Participants were 308 undergraduate university students $(\mathrm{M}=67$ and $\mathrm{F}=241)$ from the Universiti Sains Malaysia, Pulau Pinang, Malaysia. The mean (and standard deviation) of age for males and females were 22.35 (3.36) and 21.85 (1.31) respectively. After their informed consent was acquired, then a demographic questionnaire and seven inventories were completed by the participants.

\subsection{Instruments}

The demographic questionnaire included items on age, gender, religion, ethnicity, major and school of studies, marital status, order of birth, number of siblings, and number of family size. The six inventories used were: (1) Chinese College Stress Scale (CCSS), (2) Positive and Negative Affect Schedule (PANAS), (3) the Multiple Perceived Social Support Scale (MPSSS), and (4) the List of Values (LOV).

Chinese College Stress Scale (CCSS). The CCSS (48) has 34 items and includes three subscales were labeled personal hassles (daily stressors, 19 items), academic hassles (learning and examination stressors, 11 items), and negative life events (both personal and academic events, 4 items). Participants' reply to all items using a scale that ranging from 1 (strongly low) to 4 (strongly high). Using Cronbach's alpha, the internal consistency estimates were .84 for the Academic Hassles subscale, .88 for Personal Hassles, and .83 for Negative Life Events. The entire 30-item CCSS had a very satisfactory internal-consistency estimate of .91. Li and Lin affirmed CCSS validity using the Rosenberg Self-Esteem Scale (49), and the Negative Affect Scale (50).

Positive and Negative Affect Schedule (PANAS). PANAS has 20 items that include a positive affect (PANAS-P; 10 items) and a negative affect (PANAS-N; 10 items), and developed with a sample of undergraduate students and 
validated with adult populations (51). Items from the PANAS-P are interested, excited, strong, enthusiastic, proud, alert, inspired, determined, attentive, and active. Items from the PANAS-N include distressed, upset, hostile, irritable, scared, jittery, afraid, ashamed, guilty, and nervous. The PANAS was developed to be a brief measure of positive and negative affects. Respondents are asked to indicate to what extent, they have experienced each specific emotion in the past week. Each item is rated on a 5-point Likert scale ranging from 1 (very slightly or not at all) to 5 (very much or extremely). There has been considerable support for the construct validity of the PANAS. Furthermore, estimates of internal consistency have been found to range from .86 to .90 for the positive affect scale, and from .84 to .97 for the negative affect scale (51).

Multidimensional Scale of Perceived Social Support (MSPSS). The MSPSS (52) is a 12-item scale developed to assess perceived social support from family, friends, and significant others. The MSPSS is rated on a 7-point Likert-type scale $(1=$ strongly disagree to $7=$ strongly agree $)$. The MSPSS produces three scores. The Cronbach's coefficient alpha values in Zimet and colleagues were .91, .87, and .85 for the significant Others, Family, and Friends subscales respectively (53). MSPSS reliability and validity confirmed in several studies (52-54).

The List of Value scale (LOV). This scale was used to measure participants' personal values (30). There are nine items and two categories comprising the LOV scale. The internal-oriented values include the sense of accomplishment, self-respect, self-fulfillment, warm relationships with others, fun and enjoyment, and excitement. The external-oriented categories comprise a sense of belonging, security, and being well-respected. Participants were requested to indicate the extent to which each item was important in their daily lives, using a scale ranging from 1 (least important to me) to 9 (most important to me). They were also required to choose only one item deemed the most important of the 9 values to them. The LOV reliability alphas of .77 and .79 were obtained for two independent samples using the same scale. The LOV instrument is more reliable in a cross-cultural setting (55). The LOV validity was demonstrated in several previous studies $(30,56)$.

\section{Results}

Initial analysis of data included a correlation coefficient that was conducted to evaluate relationships between stress, emotional regulation, positive and negative emotions, personal values and social support. This was computed among the 13 variables in an effort to assess the degree that these quantitative variables were positive and linearly related in this sample. Findings indicated that stress total scale, personal hassle, academic hassle and negative life events hassle had significant positive relationships with positive and negative emotions. In addition, total stress scale and negative life events hassle had significantly negative relationships with personal values and its subscales. However, there are significant negative relationships between total stress scale, personal hassle and perceived social support and its family and friend subscales. In addition, there is a significant negative relationship between the life events' hassles and total perceived social support, and academic hassle hadn't any significant correlations with perceived social support and its subscale. Positive emotions, society support and total perceived support were significantly positive and linearly related. Personal values and its subscales were significantly positive and linearly correlated with perceived social support and its subscales. The Bonferroni approach was used to control for type I effort across the 13 correlations, a $\mathrm{p}$ value of less than .05 was used as an indicator for significance (Table 1).

Table 1. Correlations among Perceived Stress, Positive and Negative Emotions, Personal Values, and Perceived Social Support

\begin{tabular}{|c|c|c|c|c|c|c|c|c|c|c|c|c|}
\hline Variables & \begin{tabular}{|c|} 
Personal \\
Hassle \\
\end{tabular} & $\begin{array}{c}\text { Academic } \\
\text { Hassle }\end{array}$ & \begin{tabular}{|c} 
Life event \\
Hassle \\
\end{tabular} & $\begin{array}{c}\text { Positive } \\
\text { affect }\end{array}$ & \begin{tabular}{|c|}
$\begin{array}{c}\text { Negative } \\
\text { affect }\end{array}$ \\
\end{tabular} & $\begin{array}{c}\text { Internal } \\
\text { values } \\
\end{array}$ & $\begin{array}{c}\text { External } \\
\text { values }\end{array}$ & LOV & $\begin{array}{l}\text { Family } \\
\text { support }\end{array}$ & $\begin{array}{l}\text { Friend } \\
\text { support }\end{array}$ & $\begin{array}{l}\text { Society } \\
\text { support }\end{array}$ & PSST \\
\hline Stress & $.964^{* *}$ & $.857^{* *}$ & $.803^{* *}$ & $.255^{* *}$ & $.334^{* *}$ & $-.124^{*}$ & $-.171^{* *}$ & $-.144^{*}$ & $-.130^{*}$ & $-.133^{*}$ & -.116 & $-.156^{* *}$ \\
\hline Personal Hassle & & $.712^{* *}$ & $.712^{* *}$ & $.260^{* *}$ & $.317^{* *}$ & -.107 & $-.162^{* *}$ & $-.130^{*}$ & $-.124^{*}$ & $-.135^{*}$ & -.102 & $-.147^{*}$ \\
\hline Academic Hassle & & & $.618^{* *}$ & $.170^{* *}$ & $.266^{* *}$ & -.099 & $-.126^{*}$ & -.109 & -.090 & -.081 & -.083 & -.111 \\
\hline Life event Hassle & & & & $.226^{* *}$ & $.318^{* *}$ & $-.168^{* *}$ & $-.159^{* *}$ & $-.172^{* *}$ & -.094 & -.105 & $-.157^{* *}$ & $-.144^{*}$ \\
\hline Positive affect & & & & & $.835^{* *}$ & .000 & .046 & .017 & .105 & .070 & $.150^{* *}$ & $.128^{*}$ \\
\hline Negative affect & & & & & & -.088 & -.063 & -.083 & .060 & -.060 & .061 & .024 \\
\hline Internal values & & & & & & & $.810^{* *}$ & $.977^{* *}$ & $.337^{* *}$ & $.379^{* *}$ & $.448^{* *}$ & $.471^{* *}$ \\
\hline External values & & & & & & & & $.917^{* *}$ & $.372^{* *}$ & $.376^{* *}$ & $.438^{* *}$ & $.483^{* *}$ \\
\hline LOV & & & & & & & & & $.368^{* *}$ & $.395^{* *}$ & $.465^{* *}$ & $.498^{* *}$ \\
\hline Family support & & & & & & & & & & $.403^{* *}$ & $.495^{* *}$ & $.763^{* *}$ \\
\hline Friend support & & & & & & & & & & & $.609^{* *}$ & $.819^{* *}$ \\
\hline Society support & & & & & & & & & & & & $.874^{* *}$ \\
\hline
\end{tabular}


The second hypothesis of this research study is that gender, marital status, religion and ethnicity play a significant rolee in the stress. A t-test for independent groups was behaved to evaluate the effects of gender, and two ANOVAs were calculated for bothreligion and ethnicity differences in aforementioned independent variables. A pair of t-test for independent groups was conducted to evaluate the effects of gender and marital status, and two ANOVAs were computed for bothreligion and ethnicity differences in aforesaid independent variables. Findings rejected the effects of gender and marital status in perceived stress.

There are a significant religion differences in the personal hassle $(\mathrm{F} 2,294=5.30, \mathrm{p}=.005)$, negative life events hassle $(\mathrm{F} 2,303=5.08, \mathrm{p}=.007)$, and total stress scale $(\mathrm{F} 2,283=4.28$, $\mathrm{p}=.01$ ). Posteriori following test for group differences by Duncan's test indicated that Christian and Muslims groups had significant higher personal and negative life events' hassles and total perceived stress than Buddhist group. There are a significant ethnicity differences in the personal hassle ( $\mathrm{F} 2,240=5.31, \mathrm{p}=.006)$, negative life events hassle ( $\mathrm{F} 2$, $249=5.47, \mathrm{p}=.005)$, and total stress scale $(\mathrm{F} 2,234=4.59$, $\mathrm{p}=.01$ ). Posteriori following test for group differences by Duncan's test indicated that Malay and Others participants had significant higher personal and negative life events hassle and total stress than Chinese ethnic.

Additionally, to examine possible gender differences and age groups' interaction, a multivariate analysis of variance (MANOVA) administered by gender, marital status, ethnicity and religion variables and their interactions as independents and perceives stress and its three subscales as dependent variables. An overall multivariate effects found for gender (Wilks' $\mathrm{k}=.982 ; \mathrm{F}(3,224)=1.40 ; \mathrm{p}<.24)$ and marital status (Wilks' $\mathrm{k}=.982 ; \mathrm{F}(3,224)=1.35 ; \mathrm{p}<.25)$ perceived stress and its subscales which rejects their significant roles. Moreover, ethnicity (Wilks' $\mathrm{k}=.982 ; \mathrm{F}(6$, $448)=.685 ; \mathrm{p}<.66$ ) and religion effects (Wilks' $\mathrm{k}=.977 ; \mathrm{F}$ $(6,448)=.880 ; \mathrm{p}<.50)$ rejected in stress and its subscales.

Table 2. Predictors for Perceived Stress in the Total Sample and by Gender

\begin{tabular}{ccccccc}
\hline \multirow{2}{*}{$\begin{array}{c}\text { Total } \\
\text { sample }\end{array}$} & Predictors & $R$ & $R^{2}$ & $\beta$ & $t$ & $p$ \\
\cline { 2 - 7 } & $\begin{array}{c}\text { Negative } \\
\text { emotions }\end{array}$ & .333 & .111 & .333 & 5.67 & .001 \\
\hline \multirow{2}{*}{ Negative emo- } & $\begin{array}{c}\text { tions Perceived } \\
\text { social support }\end{array}$ & .379 & .143 & $\begin{array}{c}.339 \\
-.180\end{array}$ & $\begin{array}{c}5.87 \\
-3.12\end{array}$ & $\begin{array}{c}.001 \\
.002\end{array}$ \\
\hline \multirow{5}{*}{ Female } & $\begin{array}{c}\text { Negative } \\
\text { emotions }\end{array}$ & .487 & .237 & .487 & 4.13 & .001 \\
\cline { 2 - 7 } & $\begin{array}{c}\text { Negative } \\
\text { emotions }\end{array}$ & .287 & .083 & .287 & 4.25 & .001 \\
\cline { 2 - 7 } & $\begin{array}{c}\text { Negative emo- } \\
\text { tions Perceived } \\
\text { social support }\end{array}$ & .362 & .131 & $\begin{array}{c}.287 \\
-.221\end{array}$ & $\begin{array}{c}4.50 \\
-3.34\end{array}$ & .001 \\
.001
\end{tabular}

Finally, multiple regression analyses were conducted to evaluate positive and negative emotions, personal values and perceived social support ( 9 factors) roles in perceived stress prediction in total sample, males and females. Findings indicated that negative emotions and perceived social support explained 37 and 39 percents of perceived stress variation in total sample and females respectively, and negative emotions explained 48 percent of perceived stress among males (Table 2).

\section{Discussion}

The outcomes from this study in first hypothesis showed that stress total scale, personal, academic and negative life events hassles had significantly positive relationships with both positive and negative emotions. This result is in passage with preceding investigations, which suggested a positive relationship between stress and emotional problems (20-22, 25). As Fredrickson noted in broaden-and-build theory, it expected to be a significant relationship between the perceived stress and positive and negative emotions (22). When stress combined with the negative emotions it can be obviously relevant to survival and general adaptation. While it associated with the positive emotions it can expand personal cognition, roles repertoire, potential behavioral options and behavioral tendencies toward a useful and an efficient performance in a challenging environment, and eventually this broadened the behavioral repertoire builds skills and resources. As Salmela acclaimed previously, both negative and positive emotional affectivity against stress be able resulting to distinct outcomes with respect to their role intention and cognitive endowment (23). We hinted that experience of stress might produce either negative or positive emotions in students, but only it helped them to improve their efficacy and personal capabilities via positive emotions. For example, if students will share their stressors with others by using some emotions like interest, enjoyment, happiness, relief, affection and love, then they know how to decrease the stress perception and demonstrate their ability for a challenge against them.

In addition, present findings indicated that total stress scale and negative life events hassle had significantly negative relationships with personal values and its subscales. This finding is in concurrence with a huge of theories and literature in the personal values and their positive roles for psychological well being and the personality adjustment against distress and adverse events (26-30). We suggest personal values operate as an internal directive source which helped an individual how to manage, control and pass the stressful situations with success style.

Likewise, current findings justified significantly negative relationships between stress scale, personal hassle and perceived social support and its family and friend subscales. In addition, this study displayed a significantly negative relationship between life events' hassles and the total perceived social support. Therefore, in file with former evidence this is beneficial for the university students as well to draw support from several sources $(33,47)$. Moreover, in harmony with O'Donovan and Hughes this study affirmed that students' academic hassle hadn't any significant correlations with perceived social support and its subscale, and this finding will address to students specific need for the especial support 
in the academic situation (57). In the streak with the prediction of dual-process theory students' perception toward the supportive resources depend to some extent on how much they attend to and think about the content of a supportive aid (47). Additionally, this finding well shows the functional nature of social support in a traditional society which students prevalently search for familial, friends and society support rather than professional resources but these types of support can't help them in their academic hassle.

The results from this study in second hypothesis supported the effects of religion and ethnicity but it rejected the roles of gender and marital status in the perceived stress. Here, Christian and Muslims groups had significantly higher personal and negative life events' hassles and the total perceived stress than Buddhist group, and Malay and Others ethnics had a significantly higher personal and negative life events hassle and the total stress than Chinese group. Finally, the results from the third hypothesis revealed that negative emotions and perceived social support explained 37 and 39 percents of the perceived stress variation in total sample and females respectively, and negative emotions explained 48 percent of perceived stress among males. These discrepancies are in congruency with earlier evidence regard the importance of religion and ethnicity in the perceived stress (58-60). These differences might explain in terms of emotion, attribution and social support theories because both religion and ethnicity constructs are two cultural bounded variables and hereby mediate humans' perceived stress and their coping mechanisms in the real world and this look right more in multicultural societies like Malaysia. Since every religion and ethnicity prompt distinct expressions, perceptions, practices and ideas when people encounter stress and even their specific perspective to the nature of stress and its outcomes will differ. Alternatively, both females and males would display distinct levels of emotions, values, social networks, support due to their cultural context, and engendered roles, and hereby they have slightly a few different predictors for their perceived stress. Altogether it is plausible that childhood and succeeding psychosocial experiences in emotional affectivity, personal values and social support, will influence people reactions toward stressors in the upcoming life such as university life. This might elucidate by a few sociocultural variables such as gender, ethnicity and religion which in-depth intensively work in socialization and acculturation levels and then they influence human performance in emotions, values and perceived social support fields. This assumption could submit to by various cross cultural differences in child rearing practices, and social transformations like traditionalism, modernism, and post modernity.

\section{Conclusions}

The current research adds to the psychology literature because it examined the perceives stress, positive and negative emotions, personal values and perceived social support relationships, predictors of the perceived stress, and the impacts of religion and ethnicity in the perceived stress in an Asian non-clinical sample. Yet, this study limited because it only leaned on correlation data, and the cultural conceptualization behind the present study has to test within in an experimental approach in the future and in cross cultural samples. Nevertheless, further investigation could apply both experimental and longitudinal designs for this purpose, and to examine these constructs in clinical and non-clinical populations. But whatever are the sources of this interrelatedness in the present study, they appear advantageous for clinical interventions and public training purposes in the university based psychological centers.

\section{ACKNOWLEDGEMENTS}

This work was supported by Universiti Sains Malaysia (USM) in the Post Doctorate Fellowship of Psychology (SPD050/09) which was held by Dr. Siamak khodarahimi, Clinical Psychologist PhD.

\section{REFERENCES}

[1] Auerbach SM. Stress. In: Leong FTL, editor. Encyclopedia of counseling. Los Angeles: Sage; 2008

[2] Auerbach SM, \& Gramling, S. E. Stress management: Psychological foundations. Upper Saddle River, NJ: Prentice Hall; 1998

[3] Khodarahimi SP, N. Cognitive behavior therapy and worry reduction in an outpatient with generalized anxiety disorder. Clinical Case Studies. 2010;9(1):52-63

[4] Lazarus RS, \& Folkman, S. Stress, appraisal, and coping. NY: Springer; 1984

[5] Peden AR, Rayens, M. K., Hall, L. A., \& Beebe, L. H. Preventing depression in high risk college women: A report of an 18 month follow-up. Journal of American College Health. 2001;49:299-307

[6] Kari D, \& Heiman, T. Task-oriented versus emotion-oriented coping strategies: the case of college students. 2005 [cited 201016 June];

http://www.redorbit.com/news/display/?id=141147

[7] Bazeley P, \& Thyer, S. Stressors to student nurses beginning tertiary education: An Australian study. Nurse Education Today. 1993;13:336-42

[8] Mahat G. Stress and coping: Junior baccalaureate nursing students in clinical settings. Nursing Forum. 1998;33:11-9

[9] Kirland ML. Stressors and coping strategies among successful female African American baccalaureate nursing students. Journal of Nursing Education. 1998;37:5-12

[10] Irani L, editor. Understanding gender and confidence in CS course culture. Thirty-Fifth SIGCSE Technical Symposium on Computer Science Education; 2004; NY: ACM 
[11] Mullings L, \& Wali, A. Stress and resilience: The social context of reproduction in Central Harlem. NY: Kluwer Academic/Plenum Publishers; 2001

[12] Gadzella BM, Carvalho, C. \& Masten, W.G. Differences among gender role identity groups on stress. American Journal of Psychological Research. 2008;4:1-12

[13] McDaniel CL. The effects of stress on the gender gap in information technology. Chapel Hill, NC: School of Information and Library Science of the University of North Carolina; 2005

[14] Chen H, Wong, Y.C., Ran, M.S., \& Gilson, C. Stress among Shanghai University students: The need for social work support. Journal of Social Work. 2009;9:323-44

[15] Hudd SS, Dumlao, J., Erdman-Sager, D., Murray, D., Phan, E., Soukas, N., \& Yokozuka, N. Stress at college: Effects on health habits, health status, and self-esteem. College Student Journal. 2000;34:217-27

[16] Sarafino EP, \& Ewing, M. The Hassles Assessment Scale for students in college: Measuring the frequency and unpleasantness of and dwelling on stressful events. Journal of American College Health. 1999;48:75-83

[17] Lazarus RS. Stress and emotion: A new synthesis. NY: Springer; 1999

[18] Chrousos GP, \& Gold, P. W. The concepts of stress and stress system disorders: Overview of physical and behavioral homeostasis. JAMA.267:1244 -52

[19] Lovallo WR. Stress and health: Biological and psychological interactions. Newbury Park, CA: Sage; 1997

[20] Murberg TA, \& Bru, E. Social support, negative life events and emotional problems among Norwegian adolescents. School Psychology International. 2004(25):387-403

[21] Khodarahimi S, Rasti, A., Khajehie, M., \& Sattar, R. Students' mental health: Personal and university determinants. US-China Education Review. 2009;6:31-8

[22] Fredrickson BL. What good are positive emotions? . Review of General Psychology. 1998;2:300-19

[23] Salmela M. Intentionality and feeling: Two-level account of emotional affectivity. Nordic Journal of Philosophy. 2002; $3: 56-7$

[24] Ellsworth PC, \& Smith, C. A. Shades of joy: Patterns of appraisal differentiating pleasant emotions. Cognition and Emotion. 1988;2:301-31

[25] Tooby J, \& Cosmides, L. The past explains the present: Emotional adaptations and the structure of ancestral environments. Ethology and Sociobiology. 1990;11:375-424

[26] Allport GW, Vernon, P. E., \& Lindzey, G. Study of values. Chicago: The Riverside Publishing Company; 1970

[27] Seligman C, Olson, J. \& Zanna, M. The psychology of values. Mahwah, NJ: Lawrence Erlbaum; 1996

[28] Hofstede G. Culture's Consequences: Comparing values, behaviors, institutions and organizations across nations. Beverly Hills, CA: Sage; 2001

[29] Inglehart R. Mapping global values. . Comparative Sociology. 2006;5:115-36
[30] Kahle LR. Social values and social change: Adaptation to life in America. NY: Praeger; 1983

[31] O'Reilly C, Chatman, J., \& Caldwell, D. People and organizational culture: A Q-sort approach to assessing fit. Academy of Management Journal. 1991;34:487-516

[32] Hays RB, \& Oxley, D. Social network development and functioning during a life transition. Journal of Personality and Social Psychology. 1986;50:305-13

[33] Smith T, \& Renk, K. Predictors of academic-related stress in college students: An examination of coping, social support, parenting and anxiety. NASPA Journal. 2007;44:405-31

[34] Cobb S. Social support as moderator of life stress. Psychosomatic Medicine. 1976;38:300-14

[35] Cassel J. The contribution of the social environment to host resistance. American Journal of Epidemiology. 1976;104:107-23

[36] Pavri S, \& Monda-Amaya, L. Social support in inclusive schools: Student and teacher perspectives. Exceptional Children. 2001;67:391-411

[37] Wills TA, \& Filer, M. Social networks and social support. In: Baum A, editor. Handbook of health psychology Mahwah, NJ: Erlbaum; 2001

[38] Bowlby J. Attachment and loss. NY: Basic Books; 1982

[39] Bowlby J. A secure base: Clinical applications of attachment theory. London: Routledge; 1988

[40] Mikulincer M, \& Shaver, P. R. Security-based self- representations in adulthood: Contents and processes. In: Rholes WS, editor. Adult attachment: Theory, research, and clinical implications NY: Guilford Press; 2004

[41] Mikulincer M, \& Shaver, P. R. Attachment in adulthood: Structure, dynamics, and change. NY: Guilford Press; 2007

[42] Walker ME, Wasserman, S. and Wellman, B. Statistical models for social support networks. Sociological Methods and Research. 1993;22(1):71-98

[43] Vaux A. Social Support: Theory, research, and intervention. NY: Praeger; 1988

[44] Baldwin MW. Relational schemas and the processing of social information. Psychological Bulletin. 1992;112:461-84

[45] Burleson BR, \& MacGeorge, E. L. Supportive communication. In: Knapp ML, editor. Handbook of interpersonal communication 3th ed. Thousand Oaks, CA: Sage; 2002

[46] Clark RA, MacGeorge, E. L., \& Robinson, L. Evaluation of peer comforting strategies by children and adolescents. Human Communication Research. 2008;34:319-4

[47] Burleson BR. Understanding the outcomes of supportive communication: A dual-process approach. Journal of Social and Personal Relationships. 2009;26:21-38

[48] Li H, \& Lin, C. H. The measurement of stressful events in Chinese college students. Psychology in the Schools. 2005;42:315-23

[49] Rosenberg M. Society and the adolescent self-image. Princeton, NJ: Princeton University Press; 1965 
[50] Bradburn N. The structure of psychological well-being. Chicago: Aldine; 1969

[51] Watson D, Clark, L. A., \& Tellegen, A. Development and validation of brief measures of positive and negative affect: The PANAS scales. Journal of Personality and Social Psychology. 1988;54:1063-70

[52] Zimet GD, Dahlem, N. W., Zimet, S. G., \& Farley, G. K. The Multidimensional Scale of Perceived Social Support. Journal of Personality Assessment. 1988;52:30-41

[53] Zimet GD, Powell, S. S., Farley, G. K., Werkman, S., \& Berkoff, K.A. Psychometric characteristics of the Multidimensional Scale of Perceived Social Support. Journal of Personality Assessment. 1990;55:610-17

[54] Canty-Mitchell JZ, G.D. Psychometric properties of the Multidimensional Scale of Perceived Social Support in urban adolescents. American Journal of Community Psychology. 2000;28:391-400

[55] Shao Y. An exploratory examination of the impact of personal values on sport consumption preferences and behaviors: A cross-cultural study 2002
[56] Kahle LR, Rose, G., \& Shoham, A. Findings of LOV throughout the world, and other evidence of cross-national consumer psychographics: Introduction. Journal of $\mathrm{Eu}-$ ro-Marketing. 1999;8:1-13

[57] O'Donovan A, \& Hughes, B. M. Social support and loneliness in college students: Effects on pulse pressure reactivity to acute stress. International Journal of Adolescent Medicine and Health. 2007;19:523-38

[58] El-Gilany A, Amr., M., \& Hammad, S. Perceived stress among male medical students in Egypt and Saudi Arabia: Effect of sociodemographic factors. Annuals of Saudi Medicine. 2998;28:442-8

[59] King M, \& Schafer, W.E. Religiosity and perceived stress: A community survey. Sociological Analysis. 1992;53(1):137-47

[60] Olley LB, Brieger, W. R., \& Olley, B. O. Perceived stress factors and coping mechanisms among mothers of children with sickle cell disease in western Nigeria. Health Education Research. 1997;12:161-70 